\title{
Individuals Higher in Eating Restraint Show Heightened Physiological Arousal to Food Images
}

\author{
Maria Isabel Barros Guinle ${ }^{1}$, Mikhal A. Yudien ${ }^{2} \&$ Catherine J. Norris ${ }^{1}$ \\ ${ }^{1}$ Department of Psychology, Swarthmore College, Swarthmore, PA, USA \\ ${ }^{2}$ Perelman School of Medicine, University of Pennsylvania, Philadelphia, PA, USA \\ Correspondence: Catherine J. Norris, Department of Psychology, Swarthmore College, 500 College Avenue, \\ Swarthmore, PA 19081, USA. Tel: 1-603-369-1788. E-mail: cnorris2@ swarthmore.edu
}

\author{
Received: March 6, $2021 \quad$ Accepted: March 31, $2021 \quad$ Online Published: April 20, 2021 \\ doi:10.5539/jfr.v10n3p11 URL: https://doi.org/10.5539/jfr.v10n3p11
}

\begin{abstract}
Individuals higher in eating restraint report feeling ambivalent (i.e., both positive and negative) about food, regardless of whether it is perceived to be unhealthy or healthy (Norris, Do, Close \& Deswert, 2019). Given that ambivalence is thought to be a highly unpleasant, unstable, and arousing state, we sought in the current study to examine whether individuals higher in eating restraint show enhanced physiological arousal toward food (but not nonfood) images. Replicating our earlier findings (Norris et al., 2019), individuals higher in eating restraint exhibited more ambivalence towards both unhealthy and healthy food (but not nonfood) images than did those lower in eating restraint. Importantly, skin conductance reactivity (SCR) toward both unhealthy and healthy food images was greater for individuals higher in eating restraint than those lower in eating restraint; there were no group differences for nonfood images. Furthermore, eating restraint scores were positively correlated with SCR toward both unhealthy and healthy food images, suggesting that more extreme restraint is associated with stronger physiological arousal. Together, our results suggest that individuals higher in eating restraint experience more ambivalence and enhanced physiological arousal toward food images regardless of their perceived health value. Implications for treating individuals with eating disorders are discussed.
\end{abstract}

Keywords: ambivalence, arousal, dieter, electrodermal activity, mixed feelings, skin conductance

\section{Introduction}

In our weight- and body-focused culture, individuals are often exposed to conflicting information about proper diets, exercise routines, and lifestyles. For example, although vegetables and fruits are generally considered part of a healthy diet - the USDA Dietary Guidelines list both as important components for healthy eating (http://www.dietaryguidelines.gov/) - some popular diet plans (e.g., the Paleo diet, diets that limit nightshades, the ketogenic diet) greatly limit or even prohibit their consumption. This conflicting information may give rise to mixed positive and negative feelings (i.e., ambivalence) toward healthy food, especially for individuals prone to close monitoring of their dietary intake. Similarly, eating foods high in sugar, fat, and carbohydrates (e.g., cakes, chocolate, french fries, burgers; "unhealthy" foods), although generally thought to be highly enjoyable, may also be associated with some negative feelings, due to increased caloric intake and guilt associated with their consumption. Indeed, we have previously shown that, in general, individuals report feeling both positive and negative about foods perceived to be unhealthy; and individuals higher in dietary restraint (i.e., those with a strong concern for dieting) also report feeling ambivalent about foods perceived to be healthy (Norris, Do, Close \& Deswert, 2019).

Our work investigating food as an ambivalent stimulus was motivated by the model of evaluative space (ESM; Cacioppo \& Berntson, 1994; Cacioppo, Gardner \& Berntson, 1997, 1999; Norris, Gollan, Berntson \& Cacioppo, 2010), which proposes that negativity and positivity are independent and are combined to guide motivation either toward (i.e., to approach) or away from (i.e., to avoid) a stimulus. The ESM argues that it is not enough to know the overall valence toward a stimulus (which ranges from extremely negative to extremely positive), but that understanding the underlying negativity and positivity can (a) reveal their co-occurrence (i.e., ambivalence), and (b) ultimately provide information about complex and conflicting feelings toward a stimulus. Indeed, our previous work not only revealed that people generally feel ambivalent toward unhealthy food, but also that the ambivalence toward healthy food observed by individuals higher in eating restraint is driven by increased 
negativity (Norris et al., 2019). The ESM also proposes that one implication of having conflicting positive and negative feelings about an attitude object is that motivation is unclear; does one approach or avoid the object in question? In the absence of clear motivational direction, the individual may behave unpredictably and/or inconsistently, or may even fail to make a clear decision to approach or avoid at all. Thus, the ESM predicts that a state of ambivalence (and its related lack of behavioral guidance) may produce a cascade of additional feelings. Ambivalence may be unpleasant, conflicting, and may even increase physiological arousal (cf. Norris et al., 2010). The current study is designed to test this latter hypothesis in addition to providing a replication of our previous findings regarding feelings of ambivalence toward food.

\subsection{Arousal \& Responses to Food Stimuli}

A number of past studies have measured arousal toward food stimuli using either direct (i.e., self-reported) or indirect (e.g., implicit, psychophysiological) measures. Notably, Foroni and colleagues (2013) amassed a large dataset of food and nonfood images (i.e., the FoodCast research image database; FRIDa), and collected normative ratings - including arousal - for each, to be used in subsequent studies. Although they do not provide statistical analyses comparing different categories of their stimuli, an inspection of arousal ratings reveals some apparent differences: images of transformed food (i.e., food that has been cooked, aggregated, or preserved by humans) were rated higher in arousal $(M=48.87, S D=11.73)$ than were images of natural foods $(M=31.46, S D$ $=11.46)$ and, somewhat surprisingly, rotten food $(M=25.56, S D=8.10)$. The range in ratings indicates that participants are able to report on their arousal level while viewing food (and nonfood) images. Similarly, using the FRIDa, Padulo and colleagues (2017) found both gender and age differences in arousal/interest ratings of food images chosen to represent different categories, including "salty-sweet" foods, as well as foods characterized by their primary component (e.g., vegetable, meat), again demonstrating that participants can report their arousal toward food images.

In both of these studies, however, it is unclear whether these self-reports of arousal are related to actual physiological arousal or rather represent some other response. In addition, self-reports are known to be prone to biases, including demand characteristics, social influences, and a desire by the individual to be perceived in a positive light. Along these lines, de Wijk and colleagues (2012) argue that conscious measures of responses to food (here, products newly introduced to the market) are poor predictors of consumer acceptance; whereas unconscious (e.g., physiological) measures may be better predictors. They measured autonomic nervous system (ANS) reactivity to liked and disliked foods using three different measures; most critical for the current study is the inclusion of skin conductance responses (SCRs), which are generally recognized to index physiological arousal. Both children and adult participants showed larger SCRs when presented with disliked versus liked foods, and SCRs were stronger when participants were asked to taste or smell disliked foods than when instructed to simply visually inspect them (de Wijk et al., 2012). Thus, disliked foods appear to increase physiological arousal more so than do liked foods, and the nature of the interaction with food targets moderated this effect.

Together, these studies suggest that participants can both report their arousal towards food images and exhibit changes in physiological reactivity towards food as a function of liking. It is important to note, however, that these studies either investigated arousal responses (a) to categories of stimuli, based on the kind of food item presented (Foroni et al., 2013) or the primary composition (Padulo et al., 2017), or (b) to generally liked versus disliked food items (de Wijk et al., 2012). To our knowledge, no previous studies have looked at arousal toward food images that elicit ambivalence.

\subsection{The Effects of Arousal on Food Consumption}

Given previous findings showing that individuals do report or otherwise exhibit increased arousal toward different categories of food stimuli, a secondary question concerns the impact of this increased arousal on consummatory behavior. In other words, does increased arousal affect food consumption or decisions about food/diet? In two studies, one conducted on 18-46 year old adults (Pliner \& Melo, 1997) and a second conducted on 7-12 year old children (Pliner \& Loewen, 2002), Pliner and colleagues manipulated arousal in the laboratory and subsequently measured willingness to taste novel foods, both by actual choice behavior (Pliner \& Melo, 1997) and self-reported willingness to try (Pliner \& Loewen, 2002). Results from both studies showed that lower arousal was associated with higher willingness to try novel foods, suggesting that enhanced arousal may impact diet by pushing behavior toward the familiar. If an individual is attempting to change their diet to become healthier, arousal may have a negative impact - higher arousal in response to food may give rise to decreased motivation to try new things. Thus, if ambivalence toward food - whether healthy or unhealthy - increases physiological arousal, especially in individuals particularly focused on their dietary intact (i.e., high concern for 
dieting; high eating restraint individuals), that arousal may subsequently impact their decisions about what to eat (especially for novel food items).

\subsection{The Current Study}

(a)

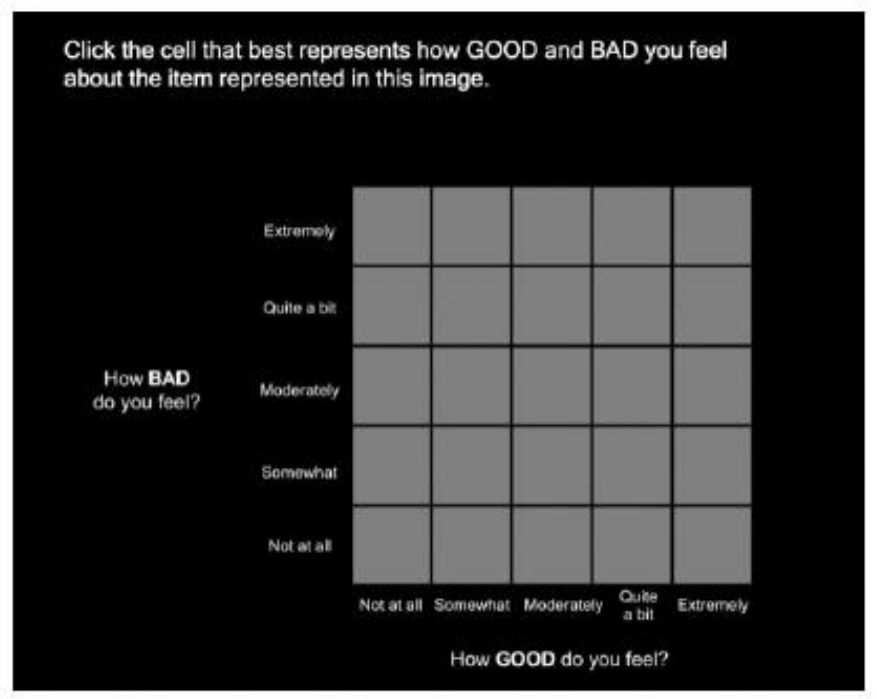

(b)

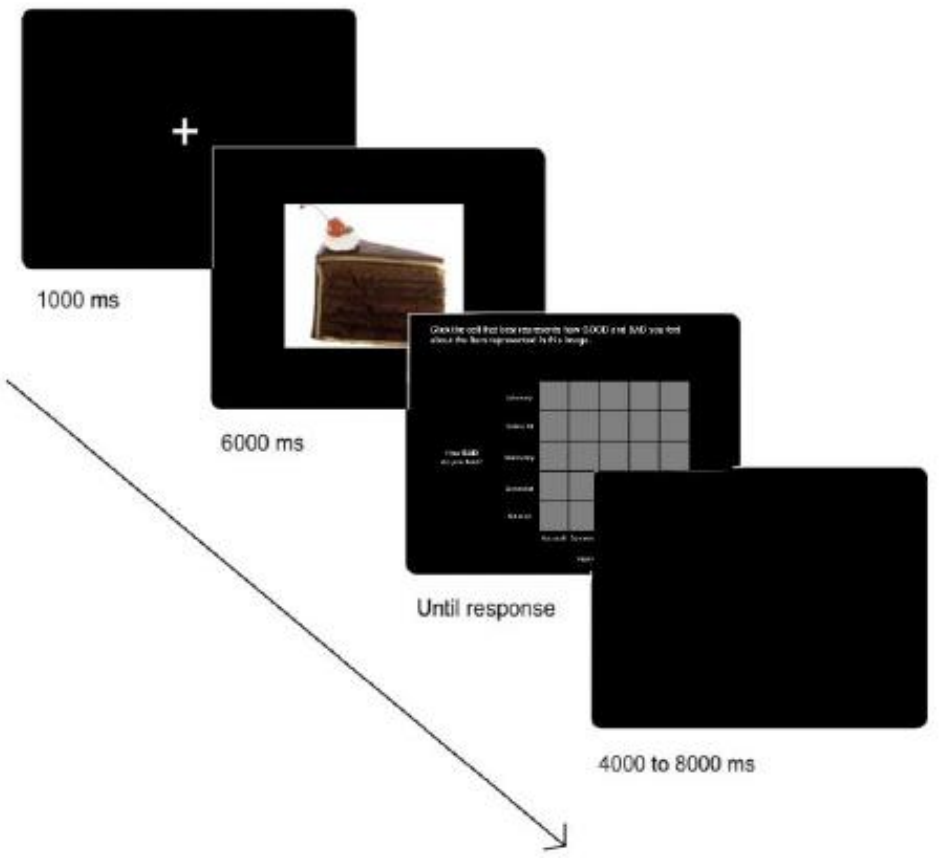

Figure 1. (a) Evaluative Space Grid (ESG). (b) Sample trial schema

Participants were presented with a fixation cross for $1000 \mathrm{~ms}$ followed by an image for $6000 \mathrm{~ms}$ and then made a rating using the ESG. Variable intertrial intervals (ITIs) followed the presentation of the ESG. Trials were self-paced and all 150 images were randomly intermixed.

In the current study, we measured physiological arousal via skin conductance reactivity, which has previously been used to assess arousal in response to visual, olfactory, and gustatory food cues (de Wijk et al., 2012, 2014), while participants viewed unhealthy food, healthy food, and nonfood images. Following each image, participants made ratings of their positive and negative feelings toward each using the Evaluative Space Grid (ESG; Larsen, Norris, McGraw, Hawkley, \& Cacioppo, 2009; Figure 1a), which allows us to also calculate measures of overall valence and ambivalence. Finally, we measured individual differences in eating restraint (i.e., chronic dieting) using the revised Eating Restraint Scale (ERS; Herman \& Mack, 1975) to examine its moderation of 
physiological and affective responses to food and nonfood images.

We first sought to replicate our past findings showing that individuals generally exhibit ambivalence (i.e., both positive and negative feelings) towards unhealthy food images, and that individuals higher in eating restraint (i.e., those with a high concern for dieting) also exhibit ambivalence towards healthy food images. We also analyzed both overall valence and independent positive and negative feelings toward food and nonfood images to better examine the complex feelings toward healthy and unhealthy food, as well as their moderation by eating restraint. Most importantly, because ambivalence is considered to be a highly arousing state, we hypothesized that individuals higher in eating restraint, who report ambivalence toward both unhealthy and healthy food images, would also show enhanced skin conductance reactivity to these images as compared to individuals lower in eating restraint. (We predicted no group differences in physiological arousal to nonfood images.) If our results support our predictions, they will contribute to a growing body of research that suggests that food is a particularly ambivalent and arousing stimulus for individuals higher in eating restraint.

\section{Method}

Although this study was not preregistered, we: (a) set a goal for sample size before conducting the study (i.e., we aimed to collect as many participants as possible between when the study was ready to begin until our cut-off date of April 15, 2019); (b) did not examine or analyze any of the data before data collection was fully complete; and (c) have uploaded all of the data to Open Science Framework (https://osf.io/tv27z/).

\subsection{Participants}

Thirty-three (16 female) (Note 1) right-handed Swarthmore College undergraduates between the ages of 18 and $21(M=19.22, S D=1.02)$ participated. Sample size was constrained by the thesis requirement at Swarthmore College; participants were run from approximately March 1, 2019 (when the study was piloted and ready for data collection) until April 15, 2019, to allow time for data analysis and writing of the thesis. Participants were compensated with either $\$ 20$ or course credit for approximately 2 hours of participation. Participants provided written and oral informed consent and the study was conducted under the supervision of the Swarthmore College Institutional Review Board.

\subsection{Procedure}

\subsubsection{Overview}

Participants received an email the day before their session that instructed them to refrain from eating for 2 hours before their scheduled session. After participants arrived at the lab and provided informed consent, they completed handedness and demographics (e.g., gender, race, and ethnicity) questionnaires, and then were asked how long it had been since they last ate and to rate their hunger on a scale from not at all hungry (1) to extremely hungry (7). Next, a respiration band was placed around the participant's torso and two electrodes were attached to the participant's non-dominant (left) palm for the collection of electrodermal activity (EDA) (Note 2). Participants were then instructed on how to use the Evaluative Space Grid (ESG; Larsen, Norris, McGraw, Hawkley, \& Cacioppo, 2009) to report how good and bad they felt about each pictured object. The ESG is a 5x5 grid with a total of 25 cells representing different degrees of positivity ( $x$-axis) and negativity ( $y$-axis; Figure 1a). Specifically, the horizontal and vertical coordinates of the ESG range from 0 (not at all good/bad) to 4 (extremely good/bad). Participants practiced moving the mouse to the cell that corresponded to the degree of positivity and negativity they were feeling for 6 different practice images ( 2 each of healthy food, unhealthy food, and nonfood) that were not repeated in experimental trials and were not used in data analysis. At this time, participants were encouraged to ask any questions of the experimenter before the task began.

\subsubsection{Food Image Viewing Task}

Following the practice trials, participants viewed 150 images (50 of each type) of healthy (Note 3) food items, unhealthy food items, or nonfood objects that were pre-selected and randomly presented. All images were selected from the FoodCast research image database (FRIDa; Foroni, Pergola, Argiris \& Rumiati, 2013) and were matched on valence, arousal, complexity, recognizability, and familiarity. Healthy and unhealthy food images were also matched on protein content, palatability, and craveability, such that any differences in response to these images were attributed to image content and not to other factors (see Norris et al., 2019 for details; the same images were used in the current study). Examples of healthy food images are fruits, vegetables, and roasted chicken; unhealthy food images are cakes, pizza, and cheeseburgers; and nonfood images are flowers, tools, and animals. On each trial, a fixation cross appeared for $1000 \mathrm{~ms}$, followed by an image for $6000 \mathrm{~ms}$. Participants then rated their responses to the image using the ESG (Larsen et al., 2009). A variable intertrial interval (4000 $8000 \mathrm{~ms}$ long) followed the ESG before the next trial began (see Figure 1b for a trial schema). 
All sensors were removed following the completion of the experimental task and participants were then seated at a desktop computer to complete a series of individual difference measures. Following these surveys, the experimenter measured the participant's height and weight (to calculate Body Mass Index; BMI) and then the participant was debriefed.

\subsubsection{Individual Differences Measures (Note 4)}

Revised Eating Restraint Scale (ERS; Herman \& Mack, 1975). The ERS consists of two subscales - concern for dieting (CD) and weight fluctuation (WF) - that assess eating restraint. The CD scale consists of 6 items, such as "Do you give too much time and thought to food?", each of which was rated on a scale ranging from almost never/not at all (1) to frequently/very much so (5). The WF scale consists of 4 items, 3 of which included items such as "What is your maximum weight gain in a week (in lb)?", that were rated on a scale ranging from 0-5 (1) to more than 26 (5). The last WF item, "In a typical week, how much does your weight fluctuate?" was rated on a scale ranging from almost never (1) to frequently (5). Although there seems to be disagreement in the literature with regard to which of the subscales is most strongly associated with disordered eating (and with eating disorder [ED] diagnosis), we made an a priori decision to focus on the $\mathrm{CD}$ subscale in the current study for the following reasons. First, theoretically, in our non-clinical sample of healthy undergraduates, we expected that concern for dieting would be more closely related to eating restraint. Second, weight fluctuation in this age group may be due to many different factors, including natural physical growth, change in diet when entering college, and stress eating at different times of the semester, among other factors. We also believed that, given the relatively young age of our participants, we would observe less (and less informative) variance in weight fluctuation; it is unlikely that our participants had had the experience of either gaining or losing extreme amounts of weight during their young lives. Thus, we focus exclusively on the CD subscale of the ERS.

\subsubsection{Skin Conductance Data Collection \& Analysis}

A respiration band was adjusted around the participant's torso and two EDA electrodes were attached to the thenar and hypothenar eminences of the participant's non-dominant (left) palm. Online, EDA was sampled at $1000 \mathrm{~Hz}$. Offline, EDA was downsampled to $250 \mathrm{~Hz}$, low-pass filtered at $15 \mathrm{~Hz}$, and visually examined for any artifacts. Baseline EDA was calculated as the average for each $1000 \mathrm{~ms}$ of the fixation cross preceding each image. Skin conductance reactivity (SCR) was calculated as the maximum value of EDA during each $6000 \mathrm{~ms}$ image presentation minus the baseline EDA for that trial (Norris, Larsen, \& Cacioppo, 2007).

\subsection{Data Analysis}

We first calculated a measure of valence for each image as the difference between the positive and negative ratings from the ESG (i.e., P - N; Larsen et al., 2009). This produces an index of valence that ranges from -4 (not at all positive/extremely negative) to 4 (extremely positive/not at all negative) which has been found to correlate with bipolar valence ratings at $r=.99$ and is the best approximation of overall feelings. Next, we calculated a measure of objective ambivalence for each image as the minimum of the positive and negative ratings from the ESG (i.e., MIN[P, N]; Kaplan, 1972). This produces an index of objective ambivalence for each image that ranges from 0 (no ambivalence) to 4 (highest ambivalence). Analyses were conducted separately on these two variables in addition to raw positive and negative ratings from the ESG. Given the normal distribution of all variables, parametric statistics were used throughout.

To examine moderation by eating restraint as measured by the Concern for Dieting subscale of the revised Eating Restraint Scale (ERS-CD), responses to the 6 ERS-CD items were averaged for each participant and then scaled such that 0 corresponded to the lowest possible score (i.e., no restraint) and 1 corresponded to the highest possible score (highest restraint). We then used a median split to categorize participants as lower or higher in ERS-CD. Scaled ERS-CD scores were relatively normally distributed $(M=0.47, S D=0.19)$, and even though our sample was drawn from a non-clinical college population, we did observe some very low (minimum $=0.10$ ) and very high $($ maximum $=0.80$ ) scores, indicating that we had sufficient range to test our hypotheses regarding individuals lower and higher in eating restraint.

Our primary analysis was a 3(image type: healthy, unhealthy, nonfood) x 2(ERS-CD: lower, higher) x 50(image) hierarchical linear model (HLM), with image type as a within-participants variable of interest, ERS-CD as a between-participants variable of interest, and image as a within-participants variable of no interest. The HLM analysis models participant variance and variance due to individual images (i.e., all data points are entered instead of simply entering an average for each condition), even though those two factors are not of interest, and is therefore a stronger test than a traditional analysis of variance (ANOVA). Importantly, because HLM uses data from each trial and partials out variance due to both individual images and participants it provides a powerful approach to analyzing this dataset, even given our somewhat small sample size (as can be seen in the degrees of 
freedom provided with each result). Thus, each HLM examines (a) the main effect of image type; (b) the main effect of ERS-CD; and (c) the interaction between image type and ERS-CD. Separate HLMs were conducted on 5 independent variables: (a) valence (i.e., P - N), (b) ambivalence (i.e., MIN[P, N]), (c) negative ratings, (d) positive ratings, and (e) skin conductance reactivity (SCR). In addition, Pearson correlations were conducted to investigate the relationship between individual differences in ERS-CD and physiological arousal. All statistical analyses were performed using the IBM SPSS program for Windows, version 24 (IBM, Chicago, IL, USA).

\section{Results}

\subsection{Self-Report Ratings of Emotional Responses to Food and Nonfood Images}

We first examined emotional responses to food and nonfood images, as well as their moderation by eating restraint, by subjecting valence, ambivalence, negative ratings, and positive ratings to separate 3(image type: healthy, unhealthy, nonfood) x 2(ERS-CD: lower, higher) x 50(image) HLMs.

\subsubsection{Valence}
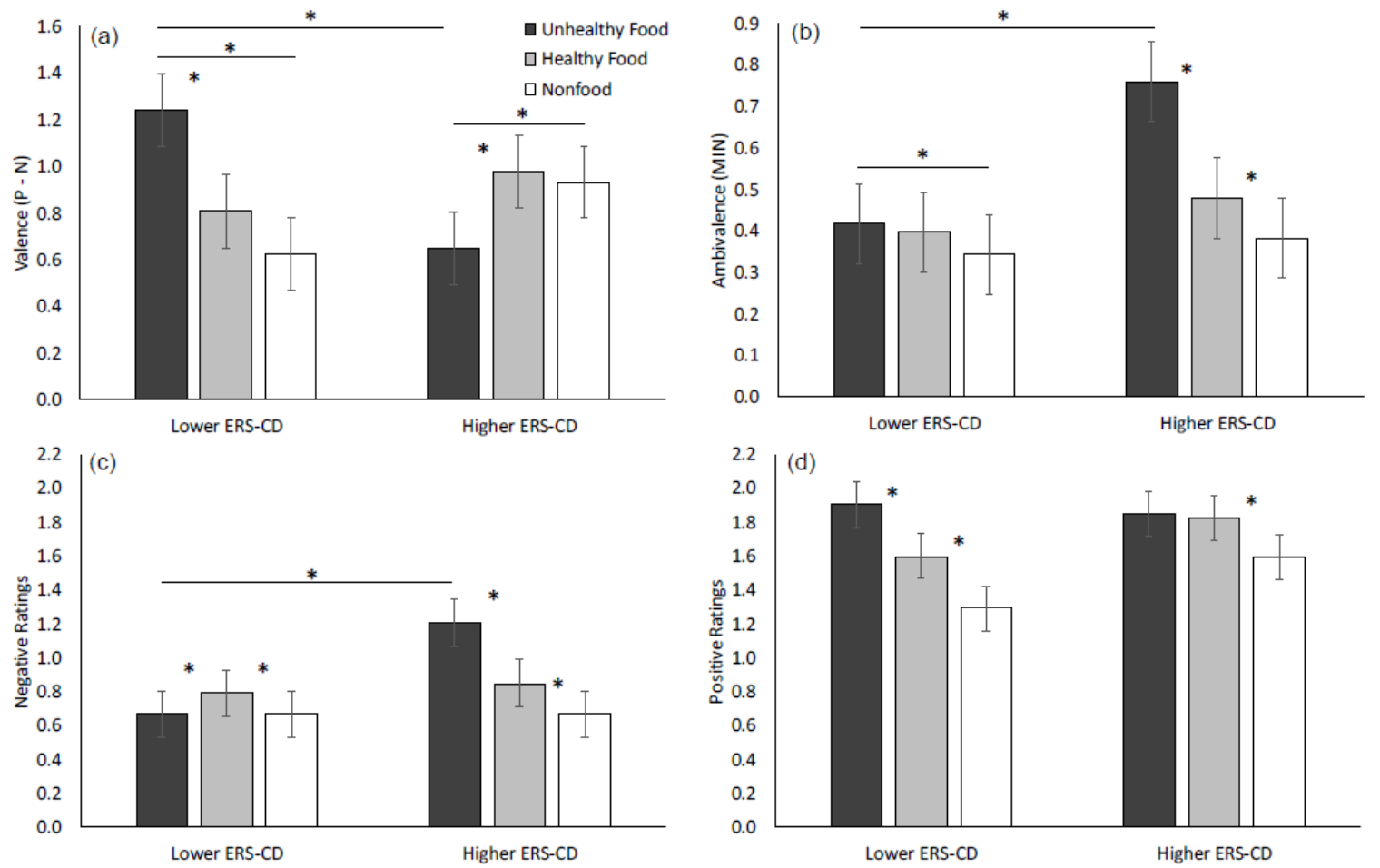

Figure 2. Image type x ERS-CD interactions for (a) valence, (b) ambivalence, (c) negative ratings, and (d) positive ratings HLMs

Although the three image categories were chosen to be matched on normative valence ratings (Foroni et al., 2013), we did find a marginal main effect of image type on valence, $F(2,3576)=3.01, p=.05$. Pairwise comparisons showed that unhealthy food images were rated as higher in valence $(M=0.94, S E=0.11)$ than were nonfood images $(M=0.78, S E=0.11), p=.017$. Neither image type differed from healthy food images $(M=$ $0.89, S E=0.11), p \mathrm{~s}>.05$. There was no main effect of ERS-CD on valence, $F(1,24)=0.03, p=.85$. However, the image type $\mathrm{x}$ ERS-CD interaction, $F(2,3576)=24.60, p<.001$, was significant (Figure 2a). Pairwise comparisons revealed that, for participants lower in ERS-CD, unhealthy food images were rated higher in valence $(M=1.24, S E=0.16)$ than healthy food images $(M=0.81, S E=0.16), p<.001$, which were only marginally higher in valence than nonfood images $(M=0.62, S E=0.16), p=.061$. For participants higher in ERS-CD, unhealthy food images were rated lower in valence $(M=0.65, S E=0.16)$ than both healthy food images $(M=0.98, S E=0.16)$ and nonfood images $(M=0.93, S E=0.16), p s<.05$; healthy food and nonfood images did not differ in valence, $p=.65$. Directly comparing valence for each image type as a function of ERS-CD, higher ERS-CD participants reported lower valence for unhealthy food images than did lower ERS-CD participants, $p=.012$; there were no group differences for healthy food or nonfood images. In sum, valence was similar for healthy food and nonfood images for participants lower and higher in ERS-CD; but lower ERS-CD 
participants reported higher valence toward unhealthy food images than the other two image types, whereas higher ERS-CD participants reported lower valence toward unhealthy food images than the other two image types.

\subsubsection{Ambivalence}

The HLM conducted on ambivalence ratings (i.e., MIN[P, N]) showed no main effect of ERS-CD on ambivalence, $F(1,24)=1.33, p=.26$. The main effect of image type, $F(2,3576)=42.93, p<.001$, revealed that participants reported more ambivalence toward unhealthy $(M=0.59, S E=0.07)$ than toward healthy $(M=0.44$, $S E=0.07)$ food images, $p<.001$, and more ambivalence toward healthy food images than toward nonfood images $(M=0.36, S E=0.07), p=.003$. This was qualified by the image type $\mathrm{x}$ ERS-CD interaction, $F(2,3576)$ $=22.09, p<.001$ (Figure 2b). Participants lower in ERS-CD reported more ambivalence toward unhealthy food images $(M=0.42, S E=0.10)$ than toward nonfood images $(M=0.34, S E=0.10), p=.037$, but showed no other differences between image types. Participants higher in ERS-CD exhibited a linear pattern, such that their responses to unhealthy food images were more ambivalent $(M=0.76, S E=0.10)$ than their responses to healthy food images $(M=0.48, S E=0.10), p<.001$, which in turn were more ambivalent than their responses to nonfood images $(M=0.38, S E=0.10), p=.006$. Again, directly comparing ambivalence between the groups for each image type, higher ERS-CD participants showed stronger ambivalence in response to unhealthy food images than did lower ERS-CD participants; there were no group differences in ambivalence toward healthy food or nonfood images. This pattern is a direct replication of our previous findings (Norris et al., 2019).

As valence and ambivalence are calculated based on raw negative and positive ratings, they provide an overall assessment of participants' affective (i.e., valence; P - N) and mixed (i.e., ambivalent; MIN[P, N]) responses to images. It is also helpful to examine raw negative and positive ratings separately to better understand the underlying mechanisms (e.g., are valence and ambivalence driven more strongly by negative or positive feelings about food?). We turn next to separate HLMs conducted on these two ratings.

\subsubsection{Negative Ratings}

The HLM conducted on negative ratings showed no main effect of ERS-CD on negative ratings, $F(1,24)=1.09$, $p=.31$. There was, however, an image type main effect, $F(2,3576)=26.73, p<.001$, such that unhealthy food images $(M=0.93, S E=0.10)$ were rated more negatively than healthy food images $(M=0.82, S E=0.10), p$ $=.002$, which in turn were rated more negatively than nonfood images $(M=0.66, S E=0.10), p<.001$. In addition, the image type $\mathrm{x}$ ERS-CD interaction was significant, $F(2,3576)=31.85, p<.001$ (Figure 2c). Pairwise comparisons revealed that, for lower ERS-CD participants, negative ratings were higher for healthy food images $(M=0.79, S E=0.14)$ than for both unhealthy food images $(M=0.66, S E=0.14), p=.016$, and for nonfood images $(M=0.67, S E=0.14), p=.018$; negative ratings for unhealthy food and nonfood images did not differ, $p=.975$. For higher ERS-CD participants, unhealthy food images were rated more negatively $(M=1.20$, $S E=0.14)$ than healthy food images $(M=0.85, S E=0.14), p<.001$, which in turn were rated more negatively than nonfood images $(M=0.66, S E=0.14), p<.001$. In other words, participants lower in eating restraint found healthy food images to be more negative than the other two image types; whereas those higher in eating restraint found both types of food images (and especially the unhealthy food images) to be more negative than nonfood images. Directly comparing the groups, higher ERS-CD participants reported higher negative ratings to unhealthy food images than did lower ERS-CD participants, $p=.01$; there were no other group differences.

\subsubsection{Positive Ratings}

There was, again, no main effect of ERS-CD on positive ratings, $F(1,24)=0.78, p=.39$. The image type main effect was significant, $F(2,3576)=7.70, p<.001$, such that unhealthy food images $(M=1.88, S E=0.09)$ were rated more positively than healthy food images $(M=1.71, S E=0.09), p=.001$, which in turn were rated more positively than nonfood images $(M=1.44, S E=0.09), p<.001$. Finally, the image type $\mathrm{x}$ ERS-CD interaction was significant, $F(2,3576)=7.70, p<.001$ (Figure 2d). Pairwise comparisons revealed that, for lower ERS-CD participants, unhealthy food images were rated more positively $(M=1.90, S E=0.13)$ than were healthy food images $(M=1.60, S E=0.13), \mathrm{p}<.001$, which in turn were rated more positively than nonfood images $(M=1.29$, $S E=0.13), p<.001$. In contrast, higher ERS-CD participants rated unhealthy $(M=1.85, S E=0.13)$ and healthy $(M=1.82, S E=0.13)$ food images as equally positive, $p=.714$; and both were more positive than nonfood images $(M=1.59, S E=0.13), p s<.001$. There were no significant group differences for positive ratings.

In sum, individuals lower in eating restraint generally felt more positive, less negative, and less ambivalent about unhealthy food in particular; whereas individuals higher in eating restraint generally felt less positive, more negative, and more ambivalent about all food, regardless of its perceived healthiness. Given that ambivalence is thought to be uncomfortable and arousing as it fails to provide behavioral guidance, the primary hypothesis of 
the current study was that higher ambivalence toward food images in individuals with higher eating restraint would result in greater levels of physiological arousal - here, measured as skin conductance reactivity - toward food versus nonfood images in high ERS-CD participants. We turn next to testing this hypothesis.

\subsection{Skin Conductance Reactivity (SCR) (Note 5)}

The HLM conducted on SCR first revealed a marginal main effect of ERS-CD, $F(1,15)=4.35, p=.055$, which showed that higher restraint participants $(M=0.26, S E=0.06)$ exhibited higher SCR than did lower restraint participants $(M=0.12, S E=0.03)$. The main effect of image type was not significant, $F(2,2235)=1.13, p=.33$. However, the image type $\mathrm{x}$ ERS-CD interaction was significant, $F(2,2235)=2.32$, one-tailed $p=.05$ (Figure 3) and, given our a priori hypothesis, we conducted follow-up tests to examine this interaction. Pairwise comparisons for participants lower in ERS-CD revealed no differences in SCR between unhealthy food $(\mathrm{M}=$ $0.12, \mathrm{SE}=0.04)$, healthy food $(\mathrm{M}=0.13, \mathrm{SE}=0.04)$, and nonfood $(\mathrm{M}=0.14, \mathrm{SE}=0.04)$ images, all $p \mathrm{~s}>.53$. For participants higher in ERS-CD, unhealthy food images elicited stronger SCR $(M=0.31, S E=0.06)$ than did nonfood images $(M=0.20, S E=0.06), p=.034$; neither image type differed from healthy food images $(M=$ $0.27, S E=0.06), p s>.23$. More importantly, however, the group comparisons revealed that higher ERS-CD participants showed stronger SCR to both unhealthy $(p=.013)$ and healthy food images $(p=.062)$ than did lower ERS-CD participants; the groups did not differ in SCR to nonfood images ( $p=.387$ ). In sum, participants higher in eating restraint showed increased physiological arousal toward both unhealthy and healthy food images as compared to low restraint participants, even though they did not differ in arousal toward nonfood images.

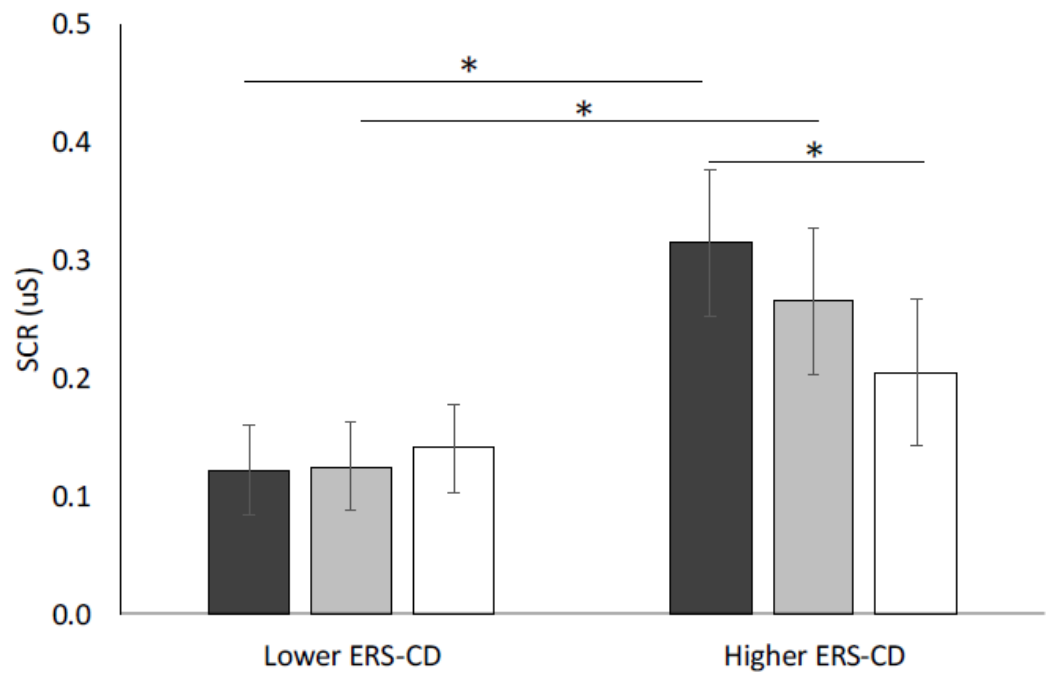

Figure 3. The image type $\mathrm{x}$ ERS-CD interaction for skin conductance reactivity. Participants lower in eating restraint showed no differences in SCR across image type; participants higher in eating restraint showed stronger

SCR to unhealthy than to healthy food images, and stronger SCR to healthy food than to nonfood images

\subsection{Correlations}

In addition to the HLM, we conducted Pearson correlations between ERS-CD $z$-scores and average SCRs (collapsed across all 50 images in each category), separately for unhealthy food, healthy food, and nonfood images. This analysis complements the HLM conducted on SCRs but more directly addresses the relationship between individual differences in eating restraint and physiological reactivity to food images. The correlations for unhealthy food images, $r(14)=.42$, one-tailed $p=.05$, and healthy food images, $r(14)=.41$, one-tailed $p$ $=.05$, were significant; the correlation for nonfood images, $r(14)=.23$, one-tailed $p=.20$, was not. Thus, higher restraint scores were associated with stronger SCR to food images, regardless of their perceived healthiness.

\section{Discussion}

\subsection{Summary}

\subsubsection{Ambivalence \& Skin Conductance}

First, results from the current study replicated our previous findings, such that, in general, individuals feel more ambivalent about unhealthy food images than about healthy food and nonfood images (Norris et al., 2019). In addition, we replicated the finding that individuals higher in eating restraint also felt more ambivalent about 
healthy food images than nonfood images, suggesting that they feel both good and bad about food, regardless of its perceived healthiness. Given that mixed emotions elicit conflict and fail to motivate behavior (either to approach or to avoid), we predicted that higher ambivalence toward food in individuals with higher eating restraint would be associated with greater physiological arousal in response to food images. Indeed, individuals higher in eating restraint showed larger skin conductance reactivity toward unhealthy food images compared to nonfood images; whereas those lower in eating restraint showed no differences in SCR across the three categories. Furthermore, SCR was larger for participants higher versus lower in eating restraint for both unhealthy and healthy food images; there were no group differences in SCR for nonfood images. This pattern demonstrates that individuals higher in eating restraint are not simply more physiologically aroused in general rather, they show heightened reactivity to food images specifically. Importantly, individual differences in eating restraint correlated with skin conductance reactivity to unhealthy and healthy food images, indicating that those with particularly high restraint (i.e., concern for dieting) showed more extreme physiological reactivity to food images, regardless of perceived healthiness. These findings are particularly important as our participants were drawn from a non-clinical sample of healthy undergraduate students, which underscores the fact that subclinical levels of eating restraint may be associated with heightened affective and physiological responses to food.

\subsubsection{Negative \& Positive Ratings}

Examining negative and positive ratings separately provided additional insight into participants' emotional responses to food images, as well as their moderation by eating restraint. Participants lower in eating restraint felt more negative about healthy food images than both nonfood and unhealthy food images; whereas those higher in eating restraint felt more negative about unhealthy food than healthy food images, and in turn more negative about healthy food than nonfood images. In particular, the two groups differed in their negativity toward unhealthy food images, with those lower in eating restraint reporting much less negativity than those higher in eating restraint. For positive ratings, participants lower in eating restraint exhibited a linear pattern, with the highest positivity toward unhealthy food, then healthy food, and finally nonfood images; whereas those higher in eating restraint showed equal positivity toward unhealthy and healthy food images, both of which were rated more positively than nonfood images. These findings are striking, given that the three image types were matched in terms of valence (i.e., P - N) using normative ratings from the FoodCast research image database (FRIDa; Foroni, Pergola, Argiris \& Rumiati, 2013). Our findings highlight the fact that using a bipolar valence scale may obscure more nuanced affective responses to food. In addition, the inclusion of nonfood images was critical for demonstrating that heightened affective and physiological responses in individuals higher in eating restraint were not simply representative of a generally stronger response disposition but rather were specific to food stimuli.

\subsection{Implications for Eating Disorders}

Although the relationship between individual differences in eating restraint and the occurrence of eating disorders (EDs) continues to be debated, it is likely that individuals with an extreme concern for dieting may be more prone to develop a dysfunctional relationship with food which could, over time, develop into a clinical ED. Although there is a dearth of existing research that has directly measured emotional and physiological responses to (healthy and unhealthy) food images in individuals with diagnosed EDs, a number of related studies are relevant to this question. Vögele and Florin (1997) examined multiple psychophysiological measures, including skin conductance reactivity, in female binge and non-binge eaters when exposed to their favorite (binge) food. They found that binge eaters showed enhanced physiological arousal (e.g., higher SCR) to binge cues than did non-binge eaters, and that binge eaters maintained these higher levels of arousal for a longer duration. Thus, there is some evidence that individuals with EDs may exhibit elevated physiological arousal in response to (relevant) food items, consistent with our own results showing that individuals higher in eating restraint also exhibit enhanced arousal to food images.

Christensen, French, and Chen (2020) measured self-reported emotional and autonomic responses to palatable food exposure in both healthy controls and women with a variety of EDs (i.e., binge-eating disorder, anorexia nervosa, bulimia nervosa) and found that participants with EDs reported greater negative affect following exposure to food as compared to healthy controls. All groups (including HCs), however, showed increased autonomic reactivity (including skin conductance) during food exposure. Increased self-reported negativity to food in those with EDs is consistent with our results showing higher negative ratings of (unhealthy) food in individuals higher in eating restraint than in those lower in eating restraint. Furthermore, Christensen et al.'s (2020) results are even more strikingly consistent with our findings when considering that the "palatable food cue" to which participants were exposed was a plate of warm chocolate chip cookies, which would fall in our unhealthy food image category. Their skin conductance results, however, are at odds with our own, as all of their 
participants showed increased SCR to the cookies and we found that only participants higher in eating restraint showed increased SCR to food images.

Similarly, one might expect that food stimuli could be particularly arousing for individuals who struggle with their weight (i.e., those who are obese). Gardner and colleagues (1988), however, found this was not the case; both obese and normal weight participants exhibited increased physiological arousal (skin conductance) during the presentation and eating of food (i.e., their favorite kind of pizza), but no group differences were observed. Thus, two previous studies have failed to find a difference in physiological arousal (i.e., SCR) to food cues as a function of EDs.

One notable difference between each of these three studies and the current study is the nature of the food cue - an image of chocolate chip cookies or pizza is unlikely to have the same effect as the actual physical presence of warm, delicious-smelling cookies (Christensen et al., 2020) or a steaming hot pizza with one's favorite toppings (Gardner et al., 1988). Even healthy controls may be physiologically aroused by the direct presence of a rewarding food stimulus. We believe this difference highlights one of the strengths of the current study; using more indirect, abstract food cues (i.e., images) may allow for a stronger test of differences in physiological reactivity to food as a function of individual differences in eating restraint (or EDs).

Another relevant study examined attentional biases toward food and body stimuli using modified Stroop paradigms, in which participants (i.e., healthy controls, patients with anorexia, and patients with bulimia) read the colors of ink in which food- and body-related words were presented (Perpiña, Leonard, Treasure, Bond, \& Baños, 1998). Patients with anorexia were slower to name the color of food-related words than were HCs, suggesting greater attentional interference for food relevant stimuli in EDs; there were no differences across groups for body-related words. Skin conductance was also collected, and patients with anorexia also exhibited higher skin conductance levels (SCLs) during food-related Stroop blocks than did patients with bulimia or HCs. In sum, patients with anorexia exhibited both increased attentional bias and higher physiological arousal when (implicitly) reading food-related words. Although this study did not examine emotional (or affective) responses to food stimuli, we argue that their results are consistent with our own, as they suggest that stronger emotional responses to food-related stimuli may be associated with greater attention to those stimuli.

Taken together, we believe that our results suggest that examining affective and physiological responses to food images in individuals with diagnosed EDs may cast additional light on the mechanisms underlying those disorders, as well as how EDs may differ. Although the relationship between eating restraint (and its subscales, weight fluctuation and concern for dieting) and EDs remains unclear, there is some evidence that individuals scoring high in concern for dieting in particular are more likely to develop EDs. For example, Killen and his colleagues (1996) found that adolescent girls scoring in the highest quartile in weight concerns had the highest incidence of showing an onset of partial syndrome eating disorders over the course of 4 years. Another study found that concern for dieting was higher in individuals with bulimia nervosa (i.e., those who binge and purge) than those with binge-eating disorder (i.e., those who binge but do not purge), suggesting that it may be a useful diagnostic tool for discriminating between these groups. If this is the case, we would predict that individuals with bulimia nervosa may (a) show stronger physiological arousal to food stimuli, (b) experience more ambivalence toward healthy food stimuli, and (c) may uniquely benefit from treatments designed to reduce their negativity and increase their positivity towards healthy foods. We might also predict that concern for dieting may be particularly high in individuals with anorexia nervosa, who should show similar patterns and benefit from the same approach to treatment. Results from the Stroop study (Perpiña et al., 1998), however, suggest that individuals with bulimia and anorexia may differ in their attention toward food-related stimuli, with those with anorexia displaying particularly strong attentional biases toward food. Conducting studies similar to ours on a sample of individuals with diagnosed EDs may contribute to a better understanding of the affective and physiological mechanisms underlying these disorders, as well as lead to different treatment approaches.

\subsection{Limitations \& Strengths}

The current study has a number of limitations worth noting. First, we recruited both male and female participants but did not have enough statistical power to examine how gender might moderate the observed effects. Gallup (2005) has found that women report dieting more frequently during their lifetime than do men. Furthermore, there is a gender difference in the diagnosis of EDs in males and females (www.nationaleatingdisorders.org). For example, Keski-Rahkonen and colleagues (2007) found that, in their sample of 9,282 Americans, 0.9\% of women but only $0.3 \%$ of men had anorexia nervosa at some point during their life. These kinds of gender differences in ED prevalence have led many researchers to limit their samples to just females, which prevents the field from investigating the effects of gender. For example, consider the possibility that all individuals with 
higher concern for dieting (regardless of gender) show elevated ambivalence (and negative affect) toward food, but only females also exhibit heightened physiological arousal to food. This pattern might shed light on the specific causal role of physiological arousal in the onset of EDs. Without including both males and females in participant samples we cannot examine different patterns of responses as a function of gender. We were hoping to be able to do so in the current study, but our sample size was limited. We anticipate conducting larger studies in the future to be able to look at moderation by gender, and we encourage other researchers to also take this analytical approach.

We also recognize that many nutritionists and researchers in related fields may take issue with our categorical designation of "healthy" and "unhealthy" food images. We acknowledge that this is a false dichotomy, and that no food, in and of itself, should be considered healthy or unhealthy; all should be considered in relation to overall dietary intake and needs. We believe, however, that this categorization is appropriate in the current study for the following reasons. First, foods were characterized as "healthy" or "unhealthy" based on previously collected ratings of perceived healthiness in a large independent sample of participants. Thus, the healthy and unhealthy categories are justified based on general perception of the foods depicted. Second, the only dimensions on which these two categories differed were fat content, carbohydrates, and kilocalories, for each of which unhealthy foods were higher than healthy foods (see Norris et al., 2019); healthy and unhealthy food images were rated as equally palatable and craveable, as well as being equal in valence and arousal (see Foroni et al., 2013 for normative ratings). Finally, using these categories allowed us to look at differences in responses to foods that people generally recognize are "good for you" (e.g., cashews, fruits and vegetables, lean forms of protein) versus "bad for you" (e.g., cookies, pizza, cheeseburgers). Using these two separate categories allowed us to further probe differences in affective responses for individuals higher and lower in eating restraint, which ultimately may shed light on disordered eating. Specifically, in the current study, higher eating restraint individuals reported feeling more negative even about foods that are generally perceived to be healthy than did those lower in eating restraint. Without using this categorical approach, we may have failed to detect some of the most informative group differences, which ultimately may lead to a better treatment approach.

Furthermore, many studies that have investigated affective and physiological responses to food in individuals with EDs have used more evocative stimuli: Christensen, French \& Chen (2020) used warm, delicious, physically-present chocolate chip cookies; Gardner and colleagues (1988) used fresh pizza; and Koushiou and colleagues (2019) used film clips of an overweight female binge-eating. Although it may be argued that our food and nonfood images are weaker stimuli, we also believe that could be seen as a strength of the current study. Given the data available for the FoodCast research image database (FRIDa; Foroni et al., 2013), we have a great deal of control over the selection of these images and were therefore able to match them on everything from craveability and palatability to physical characteristics (e.g., luminance, red/green/blue values). In addition, using a wide variety of stimuli (e.g., 50 images per category) means that idiosyncratic feelings about particular foods should be less powerful in driving observed responses to overall categories of food. In other words, if a participant simply doesn't like chocolate chip cookies, that might strongly diminish the effectiveness of Christensen and colleagues' (2020) manipulation, as chocolate chip cookies were the only food stimulus used; whereas that same participant would probably like at least a few other "unhealthy" food images in our stimulus set - such as hamburgers, pizza, cake, or potato chips. In other words, being able to present a wide variety of stimuli decreases the contribution of noise due to individual differences in food preferences in our analyses. Finally, our approach also allowed us to include a set of well-matched nonfood images, which in turn provided the opportunity to examine whether individuals higher in eating restraint were generally more anxious (i.e., gave higher ratings and/or showed larger SCRs to all image types), or whether their heightened affective and physiological responses were specific to food images (which they were). In sum, we believe that the fact that we observed clear patterns of affective and physiological responses - as well as their moderation by eating restraint in the current study, which arguably used less evocative food stimuli, underscores the impact of this work.

\subsection{Future Directions}

As mentioned above, we believe that replicating and extending the current study to include a larger sample size with an equal number of males and females - to be able to examine moderation of these effects by gender will be informative for future research. The current study did not aim to examine generalizability to a broader population, but rather to demonstrate that physiological arousal can differ as a function of food type and individual differences in eating restraint. Clearly, generalizability across gender, age, and other factors needs to be investigated in future studies.

In addition, including a self-report measure of arousal might shed more light on how affective and physiological measures converge or diverge in individuals higher and lower in eating restraint. Adding other measures of 
physiological arousal (e.g., alpha activity from the electroencephalogram [EEG], which is known to reflect levels of arousal; heart rate and heart rate variability) or more indirect measures of affective responses (e.g., facial electromyography) may also shed light on underlying mechanisms. Furthermore, based on research conducted on individuals with EDs, the inclusion of a measure of attention (e.g., eye tracking, or use of a paradigm that assess covert and/or overt attention such as the dot-probe task) to food images would help us understand how affective and physiological responses relate to attentional processes. Finally, we believe that replicating the current study in a population of individuals with diagnosed EDs would help strengthen our claims about the relationship between disordered emotional responses to food and the prevalence of eating disorder symptoms.

\section{Conclusion}

In the current study we showed that healthy, young individuals who report a higher concern for dieting also show stronger ambivalence toward healthy food images, more negativity toward unhealthy food images, and stronger physiological arousal to all food images compared to well-matched nonfood stimuli. We believe that this pattern could have implications for the onset of eating disorders, and certainly that it may contribute to a complicated and potentially disordered relationship with food. Given that individuals higher in eating restraint exhibit generalized ambivalence and physiological arousal towards both unhealthy and healthy food, one might expect this effect to be even greater in individuals with eating disorders (ED). If that is the case, then treatment should consider both the positive and negative feelings that individuals have toward food. The fact that these patterns of data were observed in a healthy, non-clinical sample of individuals in response to arguably low-impact food images in a laboratory environment suggests that they may be even stronger when eating disordered individuals navigate the real world.

\section{Acknowledgements}

We thank all members of the Norris Social Neuroscience Lab at Swarthmore College for their assistance with data collection and processing - in particular Elise Cummings, whose contributions to the study design were essential. We also thank Peiyao Chen, Ph.D., whose assistance and advice were vital for the successful completion of the study, and Andrew Ward, Ph.D. for his helpful comments and suggestions, along with the Swarthmore Social Labs Group for their feedback throughout the course of this project.

\section{References}

Banks, S. J., Bellerose, J., Douglas, D., \& Jones-Gotman, M. (2012). Bilateral skin conductance responses to emotional faces. Applied Psychophysiology and Biofeedback, 37(3), 145-152. https://doi.org/10.1007/s10484-011-9177-7

Cacioppo, J. T., \& Berntson, G. G. (1994). Relationship between attitudes and evaluative space: A critical review, with emphasis on the separability of positive and negative substrates. Psychological Bulletin, 115(3), 401-423. https://doi.org/10.1037/0033-2909.115.3.401

Cacioppo, J. T., Gardner, W. L., \& Berntson, G. G. (1997). Beyond bipolar conceptualizations and measures: The case of attitudes and evaluative space. Personality and Social Psychology Review, 1(1), 3-25. https://doi.org/10.1207/s15327957pspr0101_2

Cacioppo, J. T., Gardner, W. L., \& Berntson, G. G. (1999). The affect system has parallel and integrative processing components: Form follows function. Journal of Personality and Social Psychology, 76(5), 839-855. https://doi.org/10.1037/0022-3514.76.5.839

Carver, C. S., \& White, T. L. (1994). Behavioral inhibition, behavioral activation, and affective responses to impending reward and punishment: The BIS/BAS scales. Journal of Personality and Social Psychology, 67, 319-333. https://doi.org/10.1037/0022-3514.67.2.319

Christensen, K. A., French, M. N., \& Chen, E. Y. (2020). Multi-method assessment of palatable food exposure in women with and without eating disorders. European Eating Disorders Review: the Journal of the Eating Disorders Association, 28(5), 594-602. https://doi.org/10.1002/erv.2746

Christopoulos, G. I., Uy, M. A., \& Yap, W. J. (2019). The body and the brain: measuring skin conductance responses to understand the emotional experience. Organizational Research Methods, 22(1), 394-420. https://doi.org/10.1177/1094428116681073

Critchley, H. D., Elliott, R., Mathias, C. J., \& Dolan, R. J. (2000). Neural activity relating to generation and representation of galvanic skin conductance responses: a functional magnetic resonance imaging study. Journal of Neuroscience, 20(8), 3033-3040. https://doi.org/10.1523/JNEUROSCI.20-08-03033.2000

de Wijk, R. A., He, W., Mensink, M. G. J., Verhoeven, R. H. G., \& de Graaf, C. (2014) ANS responses and facial 
expressions differentiate between the taste of commercial breakfast drinks. PLOS ONE, 9(4), e93823. https://doi.org/10.1371/journal.pone.0093823

de Wijk, R. A., Kooijman, V., Verhoeven, R. H. G., Holthuysen, N. T. E., \& De Graaf, C. (2012). Autonomic nervous system responses on and facial expressions to the sight, smell, and taste of liked and disliked foods. Food Quality and Preference, 26, 196-203. https://doi.org/10.1016/j.foodqual.2012.04.015

Foroni, F., Pergola, G., Argiris, G., \& Rumiati, R. I. (2013). The FoodCast research image database (FRIDa). Frontiers in Human Neuroscience, 7, 51. https://doi.org/10.3389/fnhum.2013.00051

Gallup, I. (2005, August 16). Six in 10 Americans Have Attempted to Lose Weight. Retrieved March 06, 2018, from http://news.gallup.com/poll/17890/six-americans-attempted-lose weight.aspx?version=print

Gardner, R. M., Urrutia, R., Morrell, J., Espinoza, T., \& Gallegos, V. (1988). Physiological arousal of obese persons to food stimuli. Perceptual and Motor Skills, 67(3), 804-806. https://doi.org/10.2466/pms.1988.67.3.804

Garner, D. M., Olmstead, M. P., \& Polivy, J. (1983). Development and validation of a multidimensional eating disorder inventory for anorexia nervosa and bulimia. International Journal of Eating Disorders, 2(2), 15-34. https://doi.org/10.1002/1098-108X(198321)2:2<15::AID-EAT2260020203>3.0.CO;2-6

Herman, C. P., \& Mack, D. (1975). Restrained and unrestrained eating. Journal of Personality, 43, 647-60. https://doi.org/10.1111/j.1467-6494.1975.tb00727.x

Kaplan, K. J. (1972). On the ambivalence-indifference problem in attitude theory and measurement: A suggested modification of the semantic differential technique. Psychological Bulletin, 77(5), 361-372. https://doi.org/10.1037/h0032590

Keski-Rahkonen, A., Hoek, H. W., Susser, E. S., Linna, M. S., Sihvola, E., Raevuori, A., Bulik, C. M., Kaprio, J., \& Rissanen, A. (2007). Epidemiology and course of anorexia nervosa in the community. The American Journal of Psychiatry, 164(8), 1259-1265. https://doi.org/10.1176/appi.ajp.2007.06081388

Killen, J. D., Taylor, C. B., Hayward, C., Haydel, K. F., Wilson, D. M., Hammer, L., Kraemer, H., Blair-Greiner, A., \& Strachowski, D. (1996). Weight concerns influence the development of eating disorders: a 4-year prospective study. Journal of Consulting and Clinical Psychology, 64(5), 936-940. https://doi.org/10.1037//0022-006X.64.5.936

Koushiou, M., Merwin, R. M., Anderson, D., \& Karekla, M. (2019). An investigation of the affective experience of females at high risk for eating disorders in general and pathology-specific contexts. Appetite, 141, 104306. https://doi.org/10.1016/j.appet.2019.05.037

Larsen, J. T., Norris, C. J., McGraw, A. P., Hawkley, L. C., \& Cacioppo, J. T. (2009). The evaluative space grid: A single-item measure of positivity and negativity. Cognition and Emotion, 23(3), 453-480. https://doi.org/10.1080/02699930801994054

Norris, C. J., Do, E., Close, E., \& Deswert, S. (2019). Ambivalence toward healthy and unhealthy food and moderation by individual differences in restrained eating. Appetite, 140, 309-317. https://doi.org/10.1016/j.appet.2019.05.033

Norris, C. J., Gollan, J., Berntson, G. G., \& Cacioppo, J. T. (2010). The current status of research on the structure of evaluative space. Biological Psychology, 84, 422-436. https://doi.org/10.1016/j.biopsycho.2010.03.011

Norris, C. J., Larsen, J. T., \& Cacioppo, J. T. (2007). Neuroticism is associated with larger and more prolonged electrodermal responses to emotionally evocative pictures. Psychophysiology, 44, 823-826. https://doi.org/10.1111/j.1469-8986.2007.00551.x

Padulo, C., Carlucci, L., Manippa, V., Marzoli, D., Saggino, A., Tommasi, L., et al. (2017). Valence, familiarity and arousal of different foods in relation to age, sex and weight. Food Quality \& Preference, 57, 104-113. https://doi.org/10.1016/j.foodqual.2016.12.010

Perpiña, C., Leonard, T., Treasure, J., Bond, A., \& Baños, R. (1998). Selective processing of food- and body-related information and autonomic arousal in patients with eating disorders. The Spanish Journal of Psychology, 1, 3-10. https://doi.org/10.1017/S1138741600005357

Pliner, P., \& Melo, N. (1997). Food neophobia in humans: Effects of manipulated arousal and individual differences in sensation seeking. Physiology \& behavior, 61(2), 331-335.

https://doi.org/10.1016/s0031-9384(96)00406-4 
Pliner, P., \& Loewen, R. (2002). The effects of manipulated arousal on children's willingness to taste novel foods. Physiology \& Behavior, 76(4-5), 551-558. https://doi.org/10.1016/s0031-9384(02)00782-5

Stunkard, A. J., \& Messick, S. (1985). The three-factor eating questionnaire to measure dietary restraint, disinhibition and hunger. Journal of Psychosomatic Research, 29(1), 71-83. https://doi.org/10.1016/0022-3999(85)90010-8

Tranel, D., \& Damasio, H. (1994). Neuroanatomical correlates of electrodermal skin conductance responses. Psychophysiology, 31(5), 427-438. https://doi.org/10.1111/j.1469-8986.1994.tb01046.x

Vögele, C., \& Florin, I. (1997). Psychophysiological responses to food exposure: an experimental study in binge eaters. The International Journal of Eating Disorders, 21(2), 147-157.

https://doi.org/10.1002/(SICI)1098-108X(199703)21:2<147::AID-EAT5>3.0.CO;2-L

\section{Notes}

Note 1. Although our sample size may seem small, a number of past studies have successfully examined skin conductance effects in samples comparable to or even smaller than our own. We direct the interested reader to: Banks, Bellerose, Douglas \& Jones-Gotman (2012); Christopoulos, Uy \& Yap (2019); Critchley, Elliott, Mathias \& Dolan (2000); and Tranel \& Damasio (1994) for examples.

Note 2. Continuous EEG was also collected throughout the task using a 64-channel EGI electrode net (www.egi.com). These data are beyond the scope of the current paper and will be reported elsewhere.

Note 3. As addressed in Norris et al. (2019), we recognize that no food item is in itself "healthy" or "unhealthy"; rather, images were chosen for these categories based on normative ratings of perceived healthiness from a pilot study. Those ratings are also available on OSF (https://osf.io/tv27z/). As expected, food items perceived to be "unhealthy" were higher in fat content, carbohydrates, and kilocalories than were food items perceived to be "healthy."

Note 4. The following additional measures were collected but were not included in current analyses, as they were beyond the scope of this paper: The Three-Factor Eating Questionnaire (TFEQ; Stunkard \& Messick, 1985), the Eating Disorder Inventory (EDI; Garner, Olmstead, \& Polivy, 1983), and the Behavioral Inhibition System and Behavioral Activation System Scales (BIS/BAS; Carver \& White, 1994).

Note 5. Ten participants were dropped from the SCR analyses due extremely low $(<1 \mathrm{mS})$ skin conductance levels; SCR was analyzed for the remaining 23 participants ( $N=12$ females).

\section{Copyrights}

Copyright for this article is retained by the author(s), with first publication rights granted to the journal.

This is an open-access article distributed under the terms and conditions of the Creative Commons Attribution license (http://creativecommons.org/licenses/by/4.0/). 